\title{
EFFECTS OF BOUNDARY CONDITIONS ON A BOSCH-TYPE INJECTION RATE METER
}

\author{
Sandor VASS ${ }^{*}$ Máté ZÖLDY \\ Dept of Automotive Technologies, Budapest University of Technology and Economics, Hungary \\ Submitted 16 October 2019; resubmitted 21 February 2020, 21 March 2020; accepted 15 June 2020; \\ first published online 1 March 2021
}

\begin{abstract}
Spread and evolution of Common Rail (CR) injection systems enable to influence injection events more efficiently than ever, while injection mass flow rate during an injection event crucially affects the combustion process. A measurement device based on the work of Bosch was set up, and measurements were made with different boundary conditions to explore the capabilities of the measurement system and to validate a detailed model of a CR injector. The main finding of this research work is, that orifice size had no noticeable effect on the measured injection rate traces, while it was stated in the original work that a small orifice is needed to terminate the measuring tube to maintain stable measurement conditions. Moreover, the backpressure level in the measuring system has a significant effect on the measured injection traces. If pressure in the measuring tube is low, gradient at the injection rate rise is lower, while if the pressure is comparable with that of a combustion chamber maximal compression pressure, measurement of higher doses is unaccomplishable due to the long pressure decrease time in the measuring tube after the end of the injection. Based on the results of the investigation, it can be stated that the Bosch-type injection rate measurement method does not give back the exact injection rate shape, a supplementing method would be necessary to calculate real nozzle flow rate.
\end{abstract}

Keywords: diesel injector, common rail injector, injection rate measurement, injection rate meter, Bosch method, effects of boundary conditions.

\section{Notations}

Abbreviations:

$\mathrm{CR}$ - common rail;

ECU - engine control unit;

ID - internal diameter;

IC - internal combustion;

SS - stainless steel.

Variables and functions:

$A$ - cross-sectional area of the tube $\left[\mathrm{m}^{2}\right]$;

$c$ - speed of sound in the medium (diesel fuel) $[\mathrm{m} / \mathrm{s}]$;

$\Phi(x)$ - standard normal distribution function of $x$;

$m$ - mass of the measuring medium $[\mathrm{kg}]$;

$n$ - sample size $[\mathrm{pc}]$;

$p$ - pressure of the measuring medium $[\mathrm{Pa}]$;

$P(x)$ - probablility function of $x$ incidence [-];

$q$ - volume of the measuring medium $\left[\mathrm{m}^{3}\right]$;

$r_{n}$ - value of the $n$-th sample;

$t$ - time [s];

$u$ - flow velocity of the measuring medium $[\mathrm{m} / \mathrm{s}]$; $x$ - set of incidents;

$\delta$ - reliability [-];

$\kappa-$ complementary probability $(\kappa=1-\pi)[-]$;

$\pi-$ probability value $[-]$;

$\varepsilon-$ accuracy $[-]$;

$\rho-$ density $\left[\mathrm{kg} / \mathrm{m}^{3}\right]$.

\section{Introduction}

Modern diesel fuel injection systems used in road vehicles have to fulfill stringent requirements owing to the newer and stricter worldwide emission standards (Zöldy 2019). Fuel quantity, timing and regulation have to be very accurate in all engine operating points. Engines are designed to treat average diesel fuel according to the standards (Zöldy 2020). Fuel injection during one engine cycle is split into $2 . . .7$ parts to maintain the desired burn rate and through this engine performance and raw emission (Hiwase et al. 2013; Agarwal et al. 2013). This flexibility in injection

${ }^{*}$ Corresponding author. E-mail: sandor.vass@gjt.bme.hu 
timing, pressure and length (Jurić et al. 2019) could only be achieved with the appearance of CR injection systems, which separate pressure generation from fuel metering (Stumpp, Ricco 1996).

The curve of injection mass flow rate plotted against time or crankshaft angle is usually referred to as the "injection rate". The integration of this plot gives the dosage, which determines the power output of the engine. Injection rate crucially affects the combustion process; this is the reason why some research effort was put in injection rate shaping (Ghaffarpour, Baranescu 1996; Benajes et al. 2005; Rottmann et al. 2009). With the ability to influence mass flow rate during an injection event, it is now more important than ever to be able to measure the mass flow rate of CR injector nozzles during an injection process.

First attempts to measure flow rate were using rotating calibrates and stroboscopic techniques, so the injections were divided into several sections, and fuel was collected into individual beakers. Later more methods were published, including the use of electrical charges conveyed by the fuel particles (Straubel 1955) and utilization of piston movement measurement caused by the injected fluid volume (Zeuch 1961). Calibration, instrumentation and use of these systems turned out to be very complicated, so they did not become popular. Bosch (1966) proposed a new system based on the measurement of the pressure wave generated by the spray injected into a length of a compressible fluid. Experiments showed that the accuracy of both the Bosch and Zeuch methods is satisfactory; they predict similar magnitude and shape of the injection rate (Bower, Foster 1991; Takamura et al. 1992), although Bosch's method is easier to use in the research field. Other works were also dealing with injection rate measurements based on pressure change (Marčič 2003; Marcic 2006), where the spray of the injector nozzle generated pressure rise in a chamber, not in a tube. A membrane with strain gauges was used to record pressure change; hence it had a similar layout to Bosch's system. It was able to measure the flow rate of the individual nozzles, because it had a separate chamber for each nozzle. It is worthwhile to mention that Marcic first worked on a measurement device based on electric charge deposits generated by fuel flow and temperature changes in the measuring electrodes (Marčič 1999). This latter device was also compared to a Bosch fuel meter. Another very similar method was proposed by Blevins and Wagner (1999); the difference to the Bosch method was a strategically placed orifice close to the injector nozzles. Although the Bosch method was accepted as a reliable measurement device, it still had problems. Arcoumanis and Baniasad (1993) pointed out that the Bosch method gives higher fuel quantity per injection than the Zeuch, while Payri et al. (2008) provided a methodology to deal with this so-called "cumulative phenomenon". Others, like Postrioti et al. (2014), used the Zeuch method for the analysis of a CR injection system and to define the exact starting time of injection.

Based on the extensive literature available for the Bosch method, this type of device was chosen to be built.
The purpose of the work was to validate a detailed model of a CR injector. Before building an actual measurement system based on Bosch (1966), a sensitivity analysis was carried out with the help of simulation tools to understand better the inner processes of the measurement method (Vass, Németh 2013). In that work, it was shown that by changing the geometrical parameters of the device, the measured injection rate would also change, although the excitation (the injection rate trace) remains the same. As an outcome, a parameter set was defined by the simulations to get accurate results for the forthcoming measurements. After this, a measurement device was set up, and measurements were made with different boundary conditions. This way, the capabilities of the measurement system could be explored, and a detailed simulation model of a CR injector could be validated. It turned out that not only the geometrical, but the boundary conditions also crucially affect the measured injection rates.

In this work, the effect of the change of the boundary conditions will be presented and analyzed, and a set of boundary condition values will be chosen to maintain accurate measurement results, which can be compared against simulation data later. By the comparison between the simulated injection rate meter values and the measurement data, one can define the exact nozzle flow rate.

\section{The Bosch method for injection rate measurement}

When injection occurs into a length of compressible fluid, it creates pressure waves. The principle of this method is to record the change of the pressure in time, from which the volume or mass flow rate of the injection event might be calculated. The concept is based on the pressure-velocity equation valid for a single pressure wave in an instationary flow pattern (Bosch 1966):

$$
p=c \cdot \rho \cdot u \text {. }
$$

If Equation (1) is combined with the continuity equation, the governing equation of the Bosch method is given (Bower, Foster 1991):

$$
\frac{d q}{d t}=\frac{A \cdot p}{c \cdot \rho} \text {. }
$$

Equation (2) shows that the instantaneous volumetric flow rate can be calculated from the cross-sectional area of the tube, the instantaneous pressure level, the speed of sound in the fluid and the density of the fluid. Equation (2) can be easily transformed to mass flow rate by multiplication with the density:

$$
\frac{d m}{d t}=\frac{A \cdot p}{c} \text {. }
$$

Equation (3) tells that instantaneous mass flow rate is proportional to the pressure and the cross-sectional area, while inversely proportional to the speed of sound. The speed of sound in a fluid is the function of compressibility and density, but because the pressure change during the 
operation of the injection rate meter is limited, the change of these factors can be neglected (Bosch 1966). Therefore the change of the mass flow rate depends only on the change in the pressure.

Figure 1 shows the Bosch injection rate meter, which consists of five main parts. It is filled with diesel fuel as a measuring medium. The first and most complicated part of the assembly is the injector mount. It contains the installation interface of the injector, the pressure transducer, the high-pressure inlet (if needed), the low-pressure outlet (if needed) and the mounting of the measuring tube. Bosch initially used a strain gauge on a reduced crosssectional part of the injector mount, but in this work, a pressure transducer is used with its adherent amplifier and data acquisition system. The measuring tube is an equally important part of the system. The ID of the tube will predetermine the pressure raise in the fluid, and its length will define the time in which the reflected pressure wave reaches the transducer. The geometric parameters together define the internal volume of the tubing, which affects the attenuation of the pressure waves (wall and internal friction of the fluid).

Pressure waves are reflected back and forth between the injector nozzle and the termination of the measuring tube, transporting fuel in and out of the tube. Fuel exits the measuring tube depending on the end forming of the tube. If the pressure wave reflected from the termination of the measuring tube would be negative, then more fuel would leave the measuring tube than the injected volume, causing unstable conditions in the system, which is not allowed. As a result, an orifice plate or a throttle valve is needed at the end of the measuring tube; thus the size of the orifice may be adjusted according to the magnitude of the injected mass.

The orifice is connected to the so-called follower or following tube and an adjustable check valve. The length of the following tube is not critical, although it has some effect on the measured rate shape (Vass, Németh 2013).

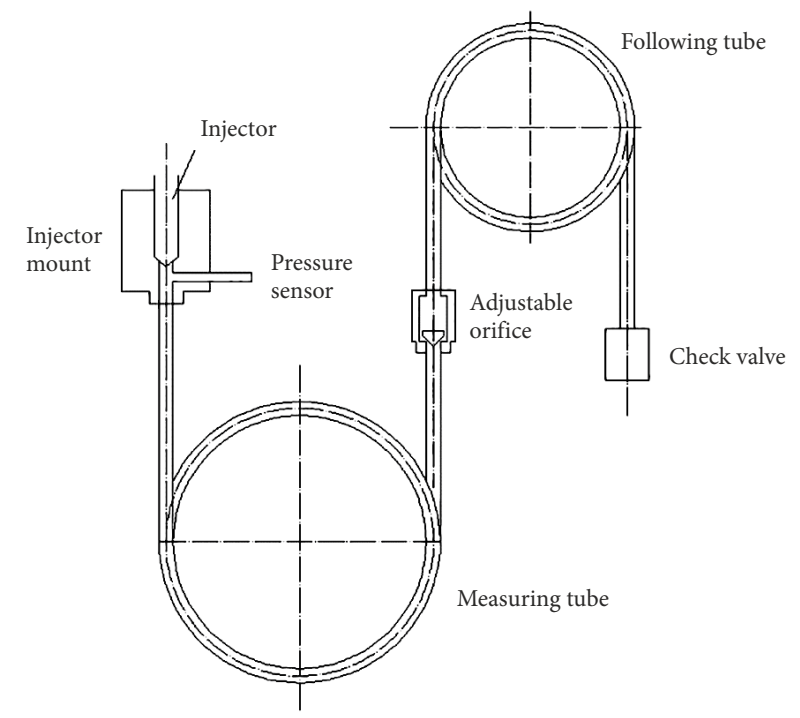

Figure 1. Schematic of the Bosch-type injection rate meter
The check valve with the following tube makes the injection observable under conditions that are similar to those in IC engines. So if the backpressure is set to the maximal compression pressure occurring in the combustion chamber of the IC engine, the pressure difference between the two sides of the orifice holes will be the same as in an engine. Also, if the ID of the measuring tube is selected to make the pressure rise in the tube comparable to the maximal combustion pressure in a given engine, then the injection process will also be similar to the one in the IC engine. The schematic of the Bosch-type injection rate meter is presented in Figure 1.

\section{Measurement setup}

In research by Vass and Németh (2013) a best tradeoff parameter set was determined based on simulations; the measuring device was built according to these values. Table 1 shows the geometric sizes of system elements; the pressure transducer was placed $20 \mathrm{~mm}$ downstream of the injector nozzle.

The injector used in the measurement was Bosch CRIN1 CR injector for commercial vehicles, information about the injector and its working principles, internal parts can be found in researches by Vass, Németh (2013) and Zöldy, Vass (2018). Fuel supply and backflow structure in commercial vehicle CR injectors is more complicated than those for passenger cars. Both the supply and backflow connection is realized inside the cylinder head, using special connecting surfaces to prevent leakage. Manufacturing such an injector mount would be complicated; hence a suitable cylinder head was used in which the injector was mounted.

The injector was connected to the high-pressure system of a four-cylinder turbocharged diesel engine installed on an engine test bench via a flexible high-pressure hose (Bárdos et al. 2014). This meant that during the measurement the engine ran with three cylinders, while the electric and hydraulic connections of the 4th cylinder were driving the injector of the Bosch meter. Therefore, injections occurred periodically in the measuring tube, which gave continuous excitation to the fuel column inside the system. A current clamp was used to record injector current and a line pressure sensor was mounted on the rail tube to measure injection pressure. The injector was equipped with a needle lift sensor measuring the control piston motion. Figure 2 shows the test bench layout.

The cylinder head was modified to accept the measuring tube from the bottom side (valve seat side) of it. The fitting made to connect the cylinder head and the

Table 1. Size of system elements

\begin{tabular}{|l|c|c|c|}
\hline \multicolumn{1}{|c|}{ Part } & ID $[\mathrm{mm}]$ & Length $[\mathrm{mm}]$ & Pressure $[\mathrm{MPa}]$ \\
\hline Measuring tube & 16 & 1500 & 5 \\
\hline Throttle valve & adjustable & - & 5 \\
\hline Following tube & 7 & 1000 & 5 \\
\hline
\end{tabular}




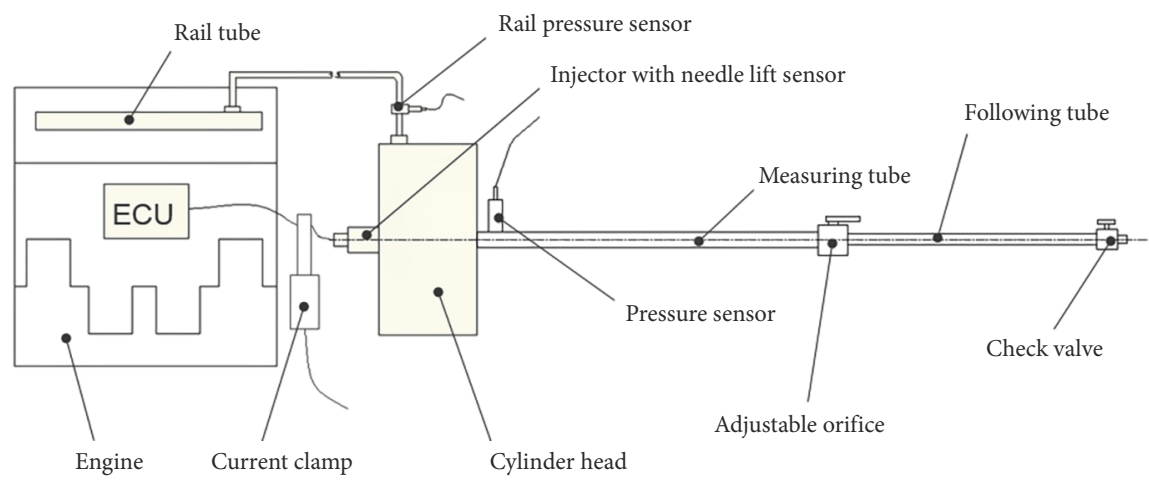

Figure 2. Test bench layout

measuring tube contained the pressure transducer, which was an AVL SL-31D-200 full bridge strain gauge sensor with $0 \ldots 20 \mathrm{MPa}$ measuring range and $100 \mathrm{kHz}$ natural frequency. The measuring and following tubes were made from 316 SS. Fittings to connect the parts were chosen to avoid ID changes. As a variable orifice, a SS quarter-turn instrument plug valve was used, while the check valve was a $316 \mathrm{SS}$ backpressure regulator, with a regulation range of $0 \ldots 13.7 \mathrm{MPa}$, equipped with a pressure gauge filled with glycerin to attenuate oscillation of the indicator.

To measure different injection pressures and excitation times, different engine operation points were set on the test bench. Table 2 presents four engine operating points, which cover injected masses from partial to full load, so that the measuring system could be tested on a wide range of excitations. All measurements were made at $1500 \mathrm{~min}^{-1}$ engine speed, except for the full load point, where the engine was not capable of a $400 \mathrm{~N} \cdot \mathrm{m}$ torque output. To make load steps consistent, the speed was lowered to 1400 $\min ^{-1}$, but this did not make any change to the results, since data was recorded as a function of time, not crankshaft position.

Engine operation points were used to compare different boundary conditions of the injection rate meter. There are two possible values to set: the size of the ori-

Table 2. Engine operating points for the injection rate measurements

\begin{tabular}{|c|c|c|c|c|}
\hline $\begin{array}{c}\text { Operation } \\
\text { point }\end{array}$ & $\begin{array}{c}\text { Engine } \\
\text { speed } \\
{\left[\mathrm{min}^{-1}\right]}\end{array}$ & $\begin{array}{c}\text { Engine } \\
\text { torque } \\
{[\mathrm{N} \cdot \mathrm{m}]}\end{array}$ & $\begin{array}{c}\text { Rail } \\
\text { pressure } \\
{[\mathrm{MPa}]}\end{array}$ & $\begin{array}{c}\text { Excitation } \\
\text { time } \\
{[\mathrm{ms}]}\end{array}$ \\
\hline 1 & 1400 & 400 & 89 & 2.7 \\
\hline 2 & 1500 & 300 & 68 & 2.55 \\
\hline 3 & 1500 & 200 & 54 & 1.9 \\
\hline 4 & 1500 & 100 & 45 & 1.15 \\
\hline
\end{tabular}

Table 3. System parts and their setting for different measurements

\begin{tabular}{|l|c|c|c|}
\hline \multicolumn{1}{|c|}{ Part } & \multicolumn{3}{c|}{ Settings } \\
\hline Plug valve $\left[^{\circ}\right]$ & 0 & 45 & 60 \\
\hline Pressure regulator $[\mathrm{MPa}]$ & 0.1 & 3 & 6 \\
\hline
\end{tabular}

fice between the measuring and following tubes, and the backpressure in the tubes. To see the effects of each, three different backpressures and orifice areas were measured for each operating point. This means that each operation point was measured with three backpressures and each backpressure with three orifice cross-sections. Altogether this means 36 measurements; for brevity, only the most characteristic traces will be presented for each boundary condition (Table 3).

\section{Results and discussion}

Each measurement was made recording a $10 \mathrm{~s}$ time segment while the engine was set to the actual operating point and boundary conditions were adjusted for the injection rate meter. The data contained a considerable amount of noise that made it impossible to select a given injection rate trace from the time interval. Different filter types were tried, but the results were not satisfying due to the phase shift and smoothing effects. The solution was to cut a time window for each injection event based on the needle lift sensor values. So, when the needle lift reached a particular value, a $3.5 \mathrm{~ms}$ window was cut from the pressure-time trace and the time segments created thus were projected on each other. In such a wise, a mean pressure value could be calculated for every time instance, which gave the pressure curve that could be used for injection rate calculation. Figure 3 shows 100 needle lifts and pressure traces that were used to calculate the average.

The needle lift profiles in Figure 3 show the deviation between individual injections, as the beginning of the needle lifts vary approximately $0.2 \mathrm{~ms}$. Sensor drift is also present, during a $10 \mathrm{~s}$ measurement zero level of the needle lift changed approximately $30 \mu \mathrm{m}$. Noise in the pressure data was an issue, but the described method gave satisfactory results, as described in the following paragraph.

To justify the number of samples in these measurements, we can use the central limit theorem. Its states that as sample size $\mathrm{n}$ gets larger, the distribution of the difference between the sample average $r_{n} / n$ and its limit $\varepsilon$, when multiplied by the factor $\sqrt{n}$, approximates the standard normal distribution. Expressed mathematically (Medvegyev 2016): 

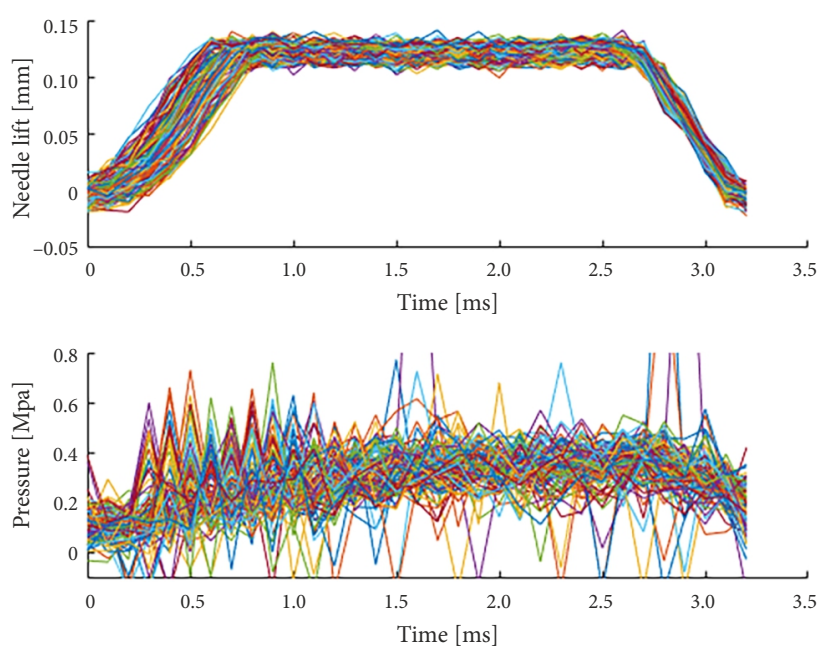

Figure 3. 100 needle lift and pressure traces for $1400 \mathrm{~min}^{-1}$, $400 \mathrm{~N} \cdot \mathrm{m}, 0^{\circ}, 0.1 \mathrm{MPa}$ measurement point

$$
\begin{aligned}
& P\left(\left|\frac{r_{n}}{n}-\pi\right| \geq \varepsilon\right)=P\left(\left|\frac{r_{n}-n \cdot \pi}{n}\right| \geq \varepsilon\right)= \\
& P\left(\left|\frac{r_{n}-n \cdot \pi}{\sqrt{n \cdot \pi \cdot \kappa}}\right| \geq \frac{\sqrt{n}}{\sqrt{\pi \cdot \kappa}} \cdot \varepsilon\right) ; \\
& P\left(|N(0,1)| \geq \frac{\sqrt{n}}{\sqrt{\pi \cdot \kappa}} \cdot \varepsilon\right) \leq P\left(|N(0,1)| \geq \frac{\sqrt{n}}{\left.\sqrt{\frac{1}{4}} \cdot \varepsilon\right)=}\right. \\
& 2 \cdot(1-\Phi(2 \cdot \sqrt{n} \cdot \varepsilon))=\delta .
\end{aligned}
$$

If we want to keep the averaged injection rate accurate, $\varepsilon$ error has to be smaller than $10 \%(\varepsilon=0.1)$. In order to have a high probability, $\delta=0.05$ will be used. Substituting these values in Equation (5):

$$
\Phi(2 \cdot \sqrt{n} \cdot \varepsilon)=1-\frac{\delta}{2}=0.975 \text {. }
$$

From this, the $n$ needed sample number can be calculated:

$$
2 \cdot \sqrt{n} \cdot \varepsilon=1.96, n=96 .
$$

Thus if more than 96 samples are used, than the calculation will have at least a $90 \%$ accuracy with more than 95\% reliability. This criterion was fulfilled, because for each calculation at least 112 measurement samples were used.

\subsection{Effect of tube pressure}

According to Bosch (1966), back pressure level would serve to adjust pressure in the fluid to match conditions of the combustion chamber. If done so, the pressure difference between the two sides of the nozzle holes of the injector is consistent with regular operation, so that the injection rate is similar to the one that would be measured in the engine. Figures 4-7 show the effect of the pressure level in the measuring tube on the injection rate measurement for all four engine operating points. Figures 4 and 5 show full and high load conditions respectively; the same effects can be observed on both. Between the $0.5 \ldots 1 \mathrm{~ms}$ time interval, the measured injection rate on $0.1 \mathrm{MPa}$ back pressure legs behind the higher pressure levels considerably. This implies that rest pressure in the system has a considerable effect on the gradient of the pressure rise in the first phase of the injection process. With higher pressures, the fluid length is "prestressed"; it has a higher bulk modulus so that pressure rise due to the injected fuel volume is steeper. The prestress of the check valve spring may have an additional effect on this process. As at $0.1 \mathrm{MPa}$ the pretension of the spring is almost zero, while at 3 and $6 \mathrm{MPa}$ it is much higher, part of the energy transmitted to the fluid is turned to open the check valve and move the closing piston against the spring in the check valve assembly.

It is worth to notice that all injection rate traces belonging to an engine operation point have very similar value at the steady-state part, where the maximal injection mass flow has been reached. This means that backpressure level does not affect the measured mass flow value during steady-state conditions, only in the transients.

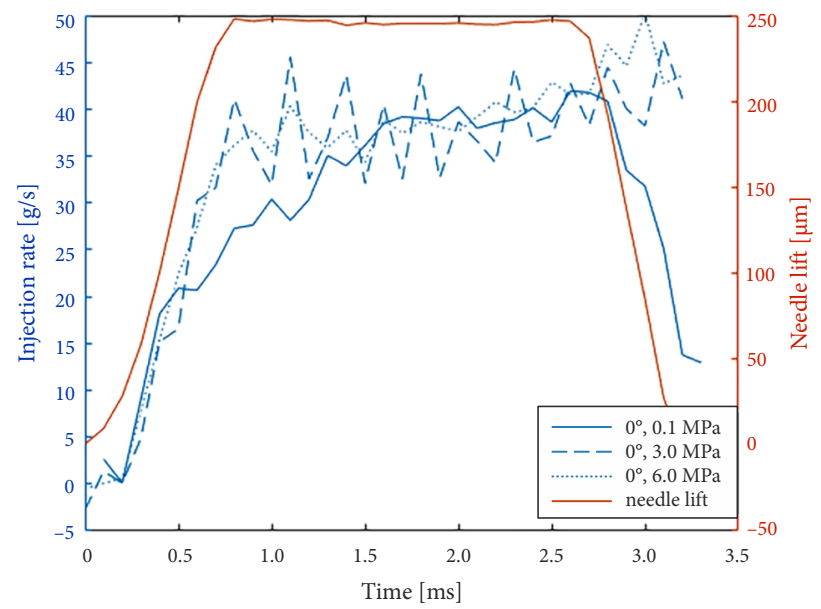

Figure 4. Measured injection rates and needle lift for $1400 \mathrm{~min}^{-1}$, $400 \mathrm{~N} \cdot \mathrm{m}, 0^{\circ}$ plug valve state, with different backpressures

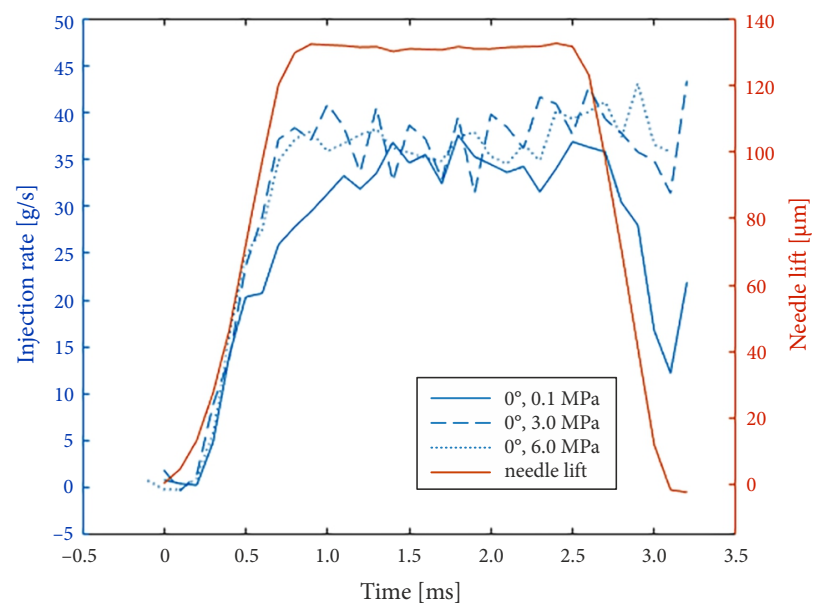

Figure 5. Measured injection rates and needle lift for $1500 \mathrm{~min}^{-1}$, $300 \mathrm{~N} \cdot \mathrm{m}, 0^{\circ}$ plug valve state, with different backpressures 


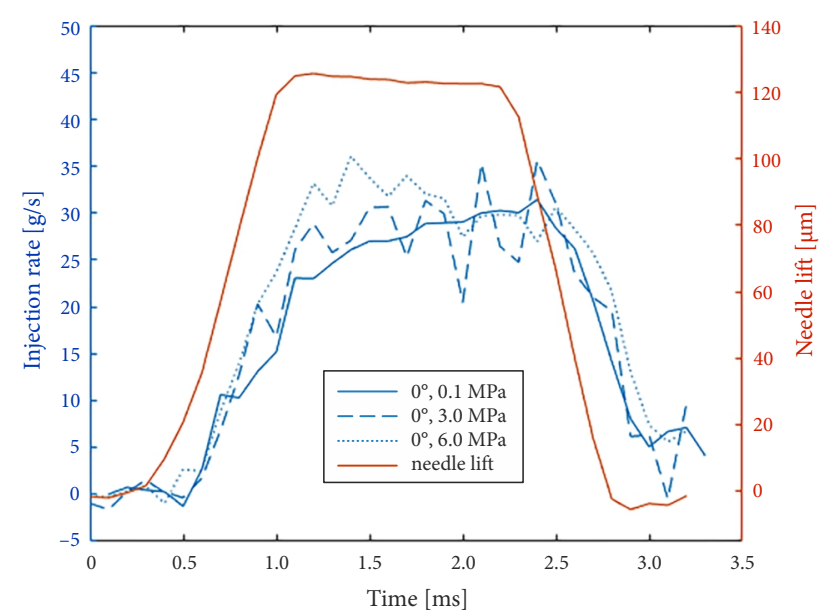

Figure 6. Measured injection rates and needle lift for $1500 \mathrm{~min}^{-1}$, $200 \mathrm{~N} \cdot \mathrm{m}, 0^{\circ}$ plug valve state, with different backpressures

The most significant differences between the different pressures appear at the end of the injections. Here at $0.1 \mathrm{MPa}$ the measurement follows the movement of the control piston of the injector closely, but at higher pressures, the measured injection rate does not drop with the closing of the needle. This is again due to system bulk modulus and the pretension of the check valve spring. As the pretension is higher and higher in the valve spring, it will act as a higher and higher choke on fluid flow during the opening (pressure reducing to rest value) phase of the operation. It is not presented in the figures, but pressure decrease to the rest value took approximately $100 \mathrm{~ms}$ between injections, which is a two orders higher time interval than injection duration. This suggests that this particular system with this particular check valve is not able to indicate the correct mass flow rate at higher injected dosage, although this inaccuracy changes on medium to low loads (Figures 6 and 7). Figure 6 shows medium engine load $(200 \mathrm{~N} \cdot \mathrm{m})$, where there is still a noticeable difference between the first, rising part of the injection rates, but it completely disappears at low load $(100 \mathrm{~N} \cdot \mathrm{m})$ and all rate traces show the same injection rate shape.

Considering every case, the $0.1 \mathrm{MPa}$ (environmental) backpressure can indicate the injection rate in all load cases, but the rising transient part of the trace is not accurate enough.

\subsection{Effect of plug valve position}

Originally Bosch terminated the measuring tube with an orifice, which reduced the flow area to a $1 . . .2 \mathrm{~mm}$ calibrated hole. It was needed to stabilize pressure waves in the tube and eliminate negative reflections, which could disturb the measurement process. In this case, an adjustable plug valve was used, which was proposed in the original work by Bosch (1966) as well.

Figures 8 and 9 illustrate the effect of three different orifice cross-sectional areas. At $0^{\circ}$ the valve was fully open, so it had the cross-sectional area of the ID $7 \mathrm{~mm}$ following tube. At $45^{\circ}$ it was reduced to half, while $60^{\circ}$ had the smallest cross-sectional area, where the system was still

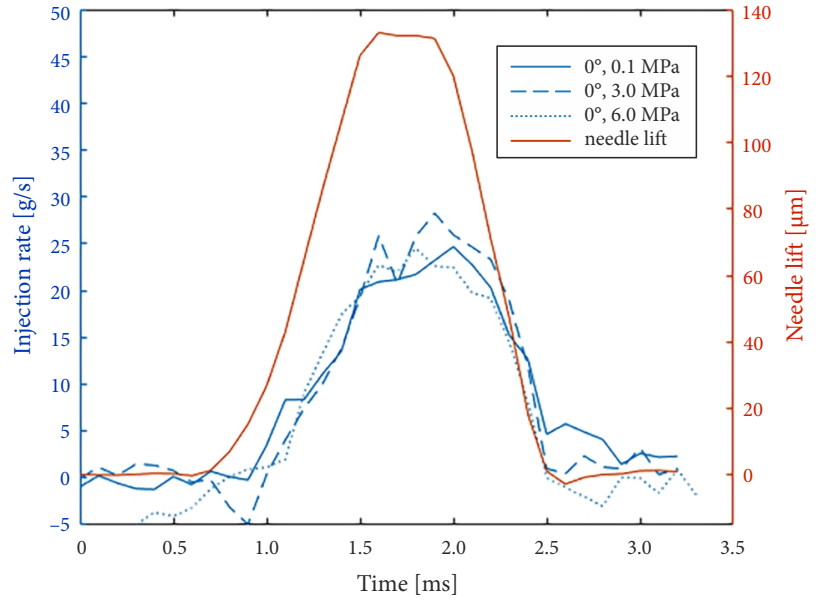

Figure 7. Measured injection rates and needle lift for $1500 \mathrm{~min}^{-1}$, $100 \mathrm{~N} \cdot \mathrm{m}, 0^{\circ}$ plug valve state, with different backpressures

stable at the highest dosages. More closing would result in excessive pressure rise due to the choking effect of the small orifice cross-sectional area.

Figure 8 shows that there is practically no effect of the orifice size on the measured injection rate. All three traces run together at the full load engine operating point; only the degressive rising of the injection rate can be observed (Payri et al. 2008), which is the result of the $0.1 \mathrm{MPa}$ backpressure. The same can be told about Figure 9, where medium engine load is illustrated with all plug valve positions.

In conclusion, it can be stated that the orifice size has no considerable effect on the measured pressure traces up until the point where the small cross-sectional area reduces the flow rate in the tube so much that it chokes the system and circumvent the measurements.

\subsection{Effect of different engine operating points}

During the measurements, engine speed was 1400 and $1500 \mathrm{~min}^{-1}$, so the excitation frequency was 11.6 and $12.5 \mathrm{~Hz}$, respectively. These excitation frequencies did not spoil the measurement data, all injections were separable and the effect of the reflections could be eliminated with the mean value calculation detailed at the beginning of Section 3. It is worth to mention here, the noise present in the measurement data was not from the oscillations, but from relatively high noise of the pressure transducer and the data acquisition system.

Figures 10 and 11 compare the different injection lengths and pressures measured with the rate meter. In Figure 10, it can be noticed that with growing injection pressure, the measured pressure gradient rises as well, while the high and full load cases (300 and $400 \mathrm{~N} \cdot \mathrm{m}$ respectively) differ almost only in the injection length. Although the measured injection rate rise is not as steep as in Figure 11 , where the $3 \mathrm{MPa}$ backpressure is presented, every injection reaches steady-state values. The 3 and $6 \mathrm{MPa}$ backpressures are not applicable for higher doses, because that makes fuel flow and pressure equalization in the system very slow. 


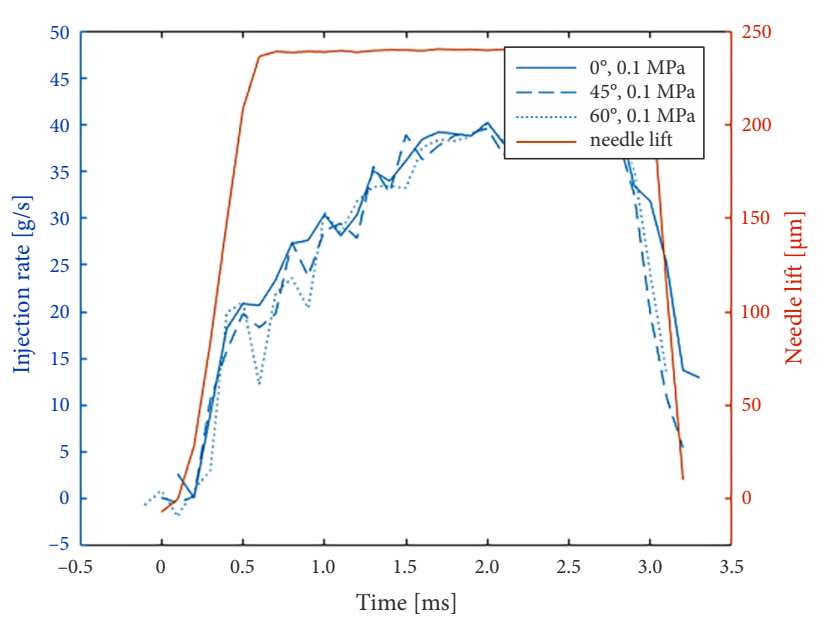

Figure 8. Measured injection rates and needle lift for $1400 \mathrm{~min}^{-1}, 400 \mathrm{~N} \cdot \mathrm{m}, 0.1 \mathrm{MPa}$ backpressure value, with different plug valve positions

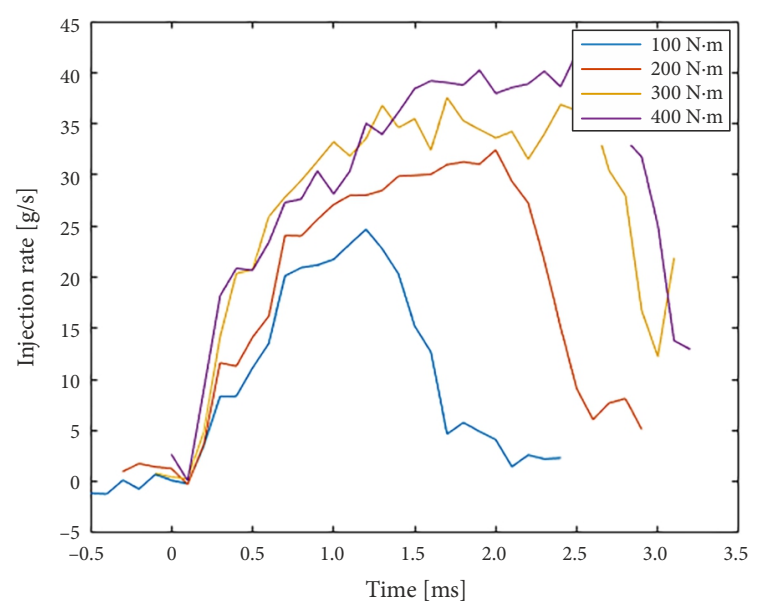

Figure 10. Measured injection rates of the four engine operating points with $0^{\circ}$ plug valve position, $0.1 \mathrm{MPa}$ backpressure

Another phenomenon can be noticed in Figures 10 and 11 - the $100 \mathrm{~N} \cdot \mathrm{m}$ load case has negative mass flow rate measured, due to the negative pressure waves reflected from the cross-sectional area change between the measuring and following tube. This is another reason why higher pressures are to be avoided in this measurement system.

\section{Conclusions}

In this work, the effect of different boundary conditions was investigated in a Bosch-type injection rate meter. Pressure and through this, injection rate shapes give useful information of the injection, although effects of the backpressure and orifice size have a considerable effect on the measured traces.

The noise of the pressure sensor and data acquisition system was eliminated with the mean value calculation of 100 consecutive injections in every corresponding time instance. This method made measurement cases comparable and the injection rate meter appropriate for the validation of a detailed CR injector model.

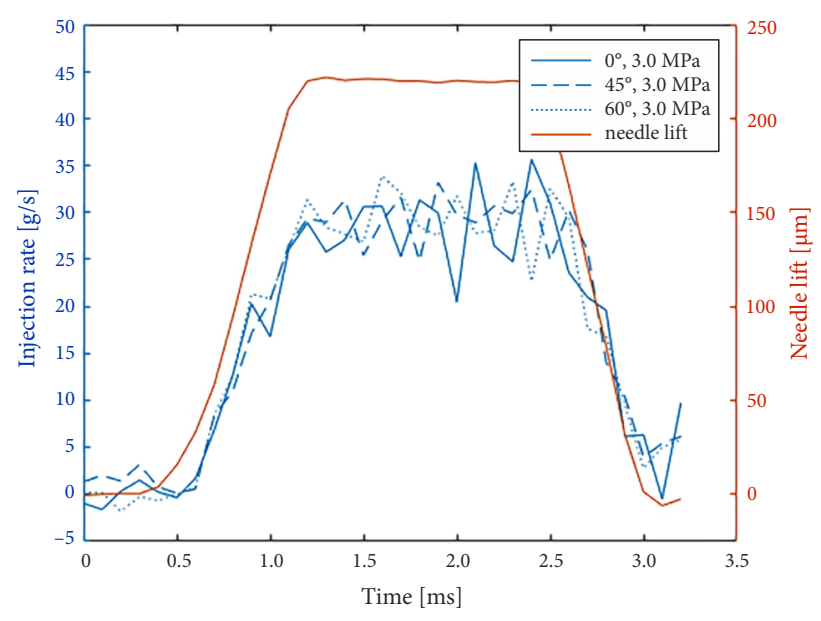

Figure 9. Measured injection rates and needle lift for $1500 \mathrm{~min}^{-1}, 200 \mathrm{~N} \cdot \mathrm{m}, 3 \mathrm{MPa}$ backpressure, with different plug valve positions

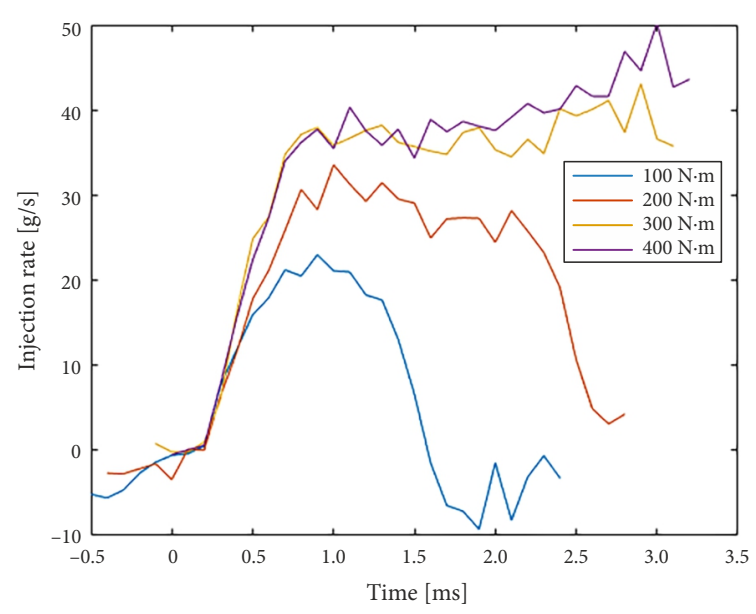

Figure 11. Measured injection rates of the four engine operating points with $0^{\circ}$ plug valve position, $6 \mathrm{MPa}$ backpressure

In the original work of Bosch, it was stated that the level of backpressure is for the setting of the pressure difference between the injector and the ambient medium to reproduce normal working conditions. Main finding of this research work is, that in this measurement system the pressure level has a significant effect on the measured injection traces: if the pressure is low, the gradient at the injection rate rise is lower; while if the pressure is comparable with that of a combustion chamber maximal compression pressure, measurement of higher doses is unaccomplishable due to the long pressure decrease time after the end of the injection.

According to our results presented here, the size of the orifice between measuring and following tubes had no noticeable effect on the measured traces, while it was stated in the original work that a small orifice is needed to terminate the measuring tube in order to maintain stable measurement conditions.

Based on the results of the investigation, a new method shall be developed for injection rate determination, because due to the change of the measured traces with differ- 
ent boundary conditions, it is not clear which one would give more reliable information about the injection event. In this new method, the correct injection rate is gathered by simulation tools, where the complete injection rate meter would be simulated together with the injector. In such a method, only the simulated injection rate meter values would have to agree with the corresponding measurement results, while the exact injection rate could be determined from the simulated injection rate of the injector itself.

\section{Author contributions}

Sandor Vass conceived the study and was responsible for the measurement system and development of the data analysis.

Sandor Vass and Máté Zöldy were responsible for data interpretation.

Sandor Vass wrote the first draft of the paper, while Máté Zöldy was responsible for the contents, suggestions and formatting, so the final paper.

\section{Disclosure statement}

The authors declare, that they do not have any competing financial, professional, or personal interests from other parties.

\section{References}

Agarwal, A. K.; Srivastava, D. K.; Dhar, A.; Maurya, R. K.; Shukla, P. C.; Singh, A. P. 2013. Effect of fuel injection timing and pressure on combustion, emissions and performance characteristics of a single cylinder diesel engine, Fuel 111: 374-383. https://doi.org/10.1016/j.fuel.2013.03.016

Arcoumanis, C.; Baniasad, M. 1993. Analysis of consecutive fuel injection rate signals obtained by the Zeuch and Bosch methods, SAE Technical Paper 930921.

https://doi.org/10.4271/930921

Bárdos, Á.; Vass, S.; Németh, H. 2014. Validation of a detailed commercial vehicle turbocharged diesel engine model, $A$ Jövö Jármüve (1-2): 25-31.

Benajes, J.; Payri, R.; Molina, S.; Soare, V. 2005. Investigation of the influence of injection rate shaping on the spray characteristics in a diesel common rail system equipped with a piston amplifier, Journal of Fluids Engineering 127(6): 1102-1110. https://doi.org/10.1115/1.2062767

Blevins, J.; Wagner, D. 1999. An experimental investigation on determining diesel injector flow and transient characteristics using high response pressure measurements, SAE Technical Paper 1999-01-0197. https://doi.org/10.4271/1999-01-0197

Bosch, W. 1966. The fuel rate indicator: a new measuring instrument for display of the characteristics of individual injection, SAE Technical Paper 660749. https://doi.org/10.4271/660749

Bower, G.; Foster, D. 1991. A comparison of the Bosch and Zuech rate of injection meters, SAE Technical Paper 910724. https://doi.org/10.4271/910724

Ghaffarpour, M.; Baranescu, R. 1996. $\mathrm{NO}_{\mathrm{x}}$ reduction using injection rate shaping and intercooling in diesel engines, $S A E$ Technical Paper 960845. https://doi.org/10.4271/960845
Hiwase, S. D.; Moorthy, S.; Prasad, H.; Dumpa, M.; Metkar, R. M. 2013. Multidimensional modeling of direct injection diesel engine with split multiple stage fuel injections, Procedia Engineering 51: 670-675. https://doi.org/10.1016/j.proeng.2013.01.095

Jurić, F.; Petranović, Z.; Vujanović, M.; Katrašnik, T.; Vihar, R.; Wang, X.; Duić, N. 2019. Experimental and numerical investigation of injection timing and rail pressure impact on combustion characteristics of a diesel engine, Energy Conversion and Management 185: 730-739.

https://doi.org/10.1016/j.enconman.2019.02.039

Marcic, M. 2006. Sensor for injection rate measurements, Sensors 6(10): 1367-1382. https://doi.org/10.3390/s6101367

Marčič, M. 1999. A new method for measuring fuel-injection rate, Flow Measurement and Instrumentation 10(3): 159-165. https://doi.org/10.1016/S0955-5986(98)00053-3

Marčič, M. 2003. Measuring method for diesel multihole injection nozzles, Sensors and Actuators A: Physical 107(2): 152158. https://doi.org/10.1016/S0924-4247(03)00205-X

Medvegyev, P. 2016. A brexit-szavazás és a nagy számok törvénye, Statisztikai Szemle 94(10): 1050-1055. (in Hungarian).

Payri, R.; Salvador, F. J.; Gimeno, J.; Bracho, G. 2008. A new methodology for correcting the signal cumulative phenomenon on injection rate measurements, Experimental Techniques 32(1): 46-49. https://doi.org/10.1111/j.1747-1567.2007.00188.x

Postrioti, L.; Buitoni, G.; Pesce, F. C.; Ciaravino, C. 2014. Zeuch method-based injection rate analysis of a common-rail system operated with advanced injection strategies, Fuel 128: 188-198. https://doi.org/10.1016/j.fuel.2014.03.006

Rottmann, M.; Menne, C.; Pischinger, S.; Luckhchoura, V.; Peters, N. 2009. Injection rate shaping investigations on a small - bore DI diesel engine, SAE Technical Paper 2009-010850. https://doi.org/10.4271/2009-01-0850

Straubel, H. 1955. Ein neuer Zerstäuber für das Flammenphotometer, Microchimica Acta 43(2-3): 329-335. https://doi.org/10.1007/BF01235004 (in German).

Stumpp, G.; Ricco, M. 1996. Common rail - an attractive fuel injection system for passenger car DI diesel engines, SAE Technical Paper 960870. https://doi.org/10.4271/960870

Takamura, A.; Ohta, T.; Fukushima, S.; Kamimoto, T. 1992. A study on precise measurement of diesel fuel injection rate, SAE Technical Paper 920630. https://doi.org/10.4271/920630

Vass, S.; Németh, H. 2013. Sensitivity analysis of instantaneous fuel injection rate determination for detailed diesel combustion models, Periodica Polytechnica Transportation Engineering 41(1): 77-85. https://doi.org/10.3311/PPtr.7106

Zeuch, W. 1961. Neue Verfahren zur Messung des Einspritzgesetztes und den Einspritz-Regelmäßigkeit von Diesel-Einspritzpumpen, MTZ 22: 344-349. (in German).

Zöldy, M. 2020. Fuel properties of butanol - hydrogenated vegetable oil blends as a diesel extender option for internal combustion engines, Periodica Polytechnica Chemical Engineering 64(2): 205-212. https://doi.org/10.3311/PPch.14153

Zöldy, M. 2019. Investigation of correlation between diesel fuel cold operability and standardized cold flow properties, Periodica Polytechnica Transportation Engineering (Online First): 1-6. https://doi.org/10.3311/PPtr.14148

Zöldy, M.; Vass, S. 2018. Detailed modelling of the internal processes of an injector for common rail systems, Journal of KONES Powertrain and Transport 25(2): 415-426. 